WU-HEP-09-04

KUNS-2222

YITP-09-49

\title{
Magnetic flux, Wilson line and orbifold
}

\author{
Hiroyuki Abe ${ }^{1, *}$, Kang-Sin $\mathrm{Choi}^{2, \dagger}$, Tatsuo Kobayashi ${ }^{2, \ddagger}$ \\ and Hiroshi Ohki ${ }^{2,3, \S}$ \\ ${ }^{1}$ Department of Physics, Waseda University, Tokyo 169-8555, Japan \\ ${ }^{2}$ Department of Physics, Kyoto University, Kyoto 606-8502, Japan \\ ${ }^{3}$ Yukawa Institute for Theoretical Physics, Kyoto University, Kyoto 606-8502, Japan
}

\begin{abstract}
We study torus/orbifold models with magnetic flux and Wilson line background. The number of zero-modes and their profiles depend on those backgrounds. That has interesting implications from the viewpoint of particle phenomenology.
\end{abstract}

*email: abe@waseda.jp

$\dagger$ †email: kschoi@gauge.scphys.kyoto-u.ac.jp

‡email: kobayash@gauge.scphys.kyoto-u.ac.jp

$\S$ email: ohki@scphys.kyoto-u.ac.jp 


\section{Introduction}

Extra dimensional field theory and string theory with magnetic fluxes can lead to interesting models [1-9]. Chiral theory can be realized as 4D effective field theory, because of magnetic flux background. The number of zero modes, that is, the generation number, is determined by the magnitude of magnetic flux. Their zero-mode profiles are non-trivially quasi-localized. Such a behavior of zero-mode wavefunctions can lead to suppressed couplings when zero-modes are quasi-localized far away each other. That would be useful to realize e.g. suppressed Yukawa couplings for light quarks and leptons. On the other hand, when their localized points are close to each other, their couplings would be of $\mathcal{O}(1)$ and that would be useful to explain e.g. the top Yukawa coupling. Furthermore, those localizing points on the torus background have a certain symmetry and it would become an origin of non-Abelian discrete flavor symmetries [10] 1] Furthermore, certain moduli can be stabilized by introducing magnetic fluxes [14]. Thus, magnetized brane models have several phenomenologically interesting aspects.

In addition, magnetized D-brane models are T-dual of intersecting D-brane models $[4-6,15-17]$. (See for a review [18] and references therein.) Within the framework of intersecting D-brane models, many interesting models have been constructed so far.

Magnetic backgrounds associated with orbifolds $[19,20]$ and Wilson lines can also derive several interesting aspects and some of them have been studied 2 Effects of Wilson lines on the torus with magnetic fluxes are gauge symmetry breaking and shift of wavefunction profiles. Orbifolding is another way to realize a chiral theory. For the same magnetic flux, the numbers of chiral zero-modes between the torus compactification and orbifold compactification are different from each other and zero-modes profiles are different $[19,20]$. Adjoint matter fields remain massless on the torus with magnetic fluxes, those are projected out on the orbifold 3 . These differences lead to phenomenologically interesting aspects [20]. However, effects due to Wilson lines have not studied on the orbifold with the magnetic flux background. Our purpose in this paper is to study more about these backgrounds such as consistency conditions, zero-mode profiles and phenomenological aspects of 4D effective theory.

This paper is organized as follows. In section 2, we study $4 \mathrm{D}$ effective theory derived from the torus compactification with magnetic flux and Wilson line background. Most of them are already known results. (See e.g. [7].) However, we reconsider phenomenological implications of Wilson lines on magnetized torus models. In section 3, we study the orbifold background with magnetic fluxes and Wilson lines. We study zero-modes under such a background and their phenomenological aspects. Section 4 is devoted to conclusion and discussion.

\footnotetext{
${ }^{1}$ Similar non-Abelian discrete flavor symmetries have been realized in heterotic orbifold models [1113].

${ }^{2}$ Other backgrounds with magnetic fluxes were also studied [21-23].

${ }^{3}$ Within the framework of intersecting D-brane models, analogous results have been obtained by considering D6-branes wrapping rigid 3-cycles [24].
} 


\section{Magnetized torus models with Wilson lines}

\section{$2.1 \quad T^{2}$ models}

Here, let us study 6D field theory with magnetic fluxes and Wilson lines. The two extra dimensions are compactified on $T^{2}$, whose area and complex structure are denoted by $A$ and $\tau$. We use the coordinates $y_{m}(m=4,5)$ for $T^{2}$, while $x_{\mu}(\mu=0, \cdots, 3)$ denote four dimensional uncompactified space-time, $R^{3,1}$. Furthermore, we often use the complex coordinate, $z=y_{4}+\tau y_{5}$. The boundary conditions on $T^{2}$ are represented by $z \sim z+1$ and $z \sim z+\tau$.

First, let us study $U(1)$ theory. We consider the fermion field $\lambda(x, z)$ with $U(1)$ charge, $q$, and it satisfies the Dirac equation,

$$
\Gamma^{M} D_{M} \lambda(x, z)=\Gamma^{M}\left(\partial_{M}-i q A_{M}\right) \lambda(x, z)=0,
$$

with $M=0, \cdots, 5$, where $\Gamma^{M}$ denote the $6 \mathrm{D}$ gamma matrices and $A_{M}$ denote $U(1)$ gauge vectors. The fermion field $\lambda$, the vector fields $A_{M}(M=(\mu, m))$ are decomposed as

$$
\begin{aligned}
\lambda(x, z) & =\sum_{n} \chi_{n}(x) \otimes \psi_{n}(z), \\
A_{\mu}(x, z) & =\sum_{n} A_{n, \mu}(x) \otimes \phi_{n, \mu}(z), \\
A_{m}(x, z) & =\sum_{n} \varphi_{n, m}(x) \otimes \phi_{n, m}(z),
\end{aligned}
$$

with $m=4,5$, where $A_{n, \mu}(x)$ and $\varphi_{n, m}(x)$ correspond to $4 \mathrm{D}$ vector fields and scalar fields, respectively. Here, the modes with $n=0$ correspond to zero-modes, while the others correspond to massive modes. Since we concentrate on zero-modes, we omit the subscript corresponding to $n=0$.

We assume the following form of magnetic flux on $T^{2}$,

$$
F=\frac{\pi i}{\operatorname{Im} \tau} m(d z \wedge d \bar{z})
$$

where $m$ is an integer [25]. Such magnetic flux can be obtained from the vector potential,

$$
A(z)=\frac{\pi m}{\operatorname{Im} \tau} \operatorname{Im}(\bar{z} d z) .
$$

This form of the vector potential satisfies the following relations,

$$
\begin{aligned}
& A(z+1)=A(z)+\frac{\pi m}{\operatorname{Im} \tau} \operatorname{Im}(d z) \\
& A(z+\tau)=A(z)+\frac{\pi m}{\operatorname{Im} \tau} \operatorname{Im}(\bar{\tau} d z) .
\end{aligned}
$$

Furthermore, these can be represented as the following gauge transformations,

$$
A(z+1)=A(z)+d \chi_{1}, \quad A(z+\tau)=A(z)+d \chi_{2},
$$


where

$$
\chi_{1}=\frac{\pi m}{\operatorname{Im} \tau} \operatorname{Im}(z), \quad \chi_{2}=\frac{\pi m}{\operatorname{Im} \tau} \operatorname{Im}(\bar{\tau} z) .
$$

Then, the fermion field $\psi(z)$ with the charge $q$ must satisfy

$$
\psi(z+1)=e^{i q \chi_{1}(z)} \psi(z), \quad \psi(z+\tau)=e^{i q \chi_{2}(z)} \psi(z) .
$$

Here and hereafter, we use the $U(1)$ charge normalization that all charges of matter fields are equal to integers and the minimum charge is $|q|=1$. The internal part $\psi$ of the fermion zero-modes is two-component spinor,

$$
\psi=\left(\begin{array}{c}
\psi_{+} \\
\psi_{-}
\end{array}\right)
$$

and we choose the gamma matrix for $T^{2}$,

$$
\tilde{\Gamma}^{1}=\left(\begin{array}{cc}
0 & 1 \\
1 & 0
\end{array}\right), \quad \tilde{\Gamma}^{2}=\left(\begin{array}{cc}
0 & -i \\
i & 0
\end{array}\right)
$$

Then, the Dirac equations for zero-modes become

$$
\begin{aligned}
& \left(\bar{\partial}+\frac{\pi q m}{2 \operatorname{Im}(\tau)} z\right) \psi_{+}(z, \bar{z})=0 \\
& \left(\partial-\frac{\pi q m}{2 \operatorname{Im}(\tau)} \bar{z}\right) \psi_{-}(z, \bar{z})=0 .
\end{aligned}
$$

When $q m>0$, the component $\psi_{+}$has $M=q m$ independent zero-modes and their wavefunctions are written as [7]

$$
\Theta^{j, M}(z)=N_{M} e^{i \pi M z \operatorname{Im}(z) / \operatorname{Im}(\tau)} \vartheta\left[\begin{array}{c}
j / M \\
0
\end{array}\right](M z, M \tau),
$$

where $N_{M}$ is a normalization factor, $j$ denotes the flavor index, i.e. $j=0, \cdots, M-1$ and

$$
\vartheta\left[\begin{array}{l}
a \\
b
\end{array}\right](\nu, \mu)=\sum_{n} \exp \left[\pi i(n+a)^{2} \mu+2 \pi i(n+a)(\nu+b)\right],
$$

that is, the Jacobi theta-function. Note that $\Theta^{0, M}(z)=\Theta^{M, M}(z)$. They satisfy the orthonormal condition,

$$
\int d^{2} z \Theta^{i, M}(z)\left(\Theta^{j, M}(z)\right)^{*}=\delta_{i j}
$$

Furthermore, for $q m>0$, the other component $\psi_{-}$has no zero-modes. As a result, we can realize a chiral spectrum.

On the other hand, when $q m<0$, the component $\psi_{-}$has $|q m|$ independent zeromodes, but the other component $\psi_{+}$has no zero-modes. 
One of important properties of zero-mode wavefunctions is that we can decompose a product of two zero-mode wavefunctions as follows [26,27],

$$
\begin{aligned}
\Theta^{i, M_{1}}(z) \Theta^{j, M_{2}}(z)= & \frac{N_{M_{1}} N_{M_{2}}}{N_{M_{1}+M_{2}}} \sum_{m \in \mathbf{Z}_{M_{1}+M_{2}}} \Theta^{i+j+M_{1} m, M_{1}+M_{2}}(z) \\
& \times \vartheta\left[\begin{array}{c}
\frac{M_{2} i-M_{1} j+M_{1} M_{2} m}{M_{1} M_{2}\left(M_{1}+M_{2}\right)} \\
0
\end{array}\right]\left(0, \tau_{d} M_{1} M_{2}\left(M_{1}+M_{2}\right)\right) .
\end{aligned}
$$

Now, let us introduce Wilson lines. The Dirac equations of the zero-modes are modified by the Wilson line background, $C=C_{1}+\tau C_{2}$ as

$$
\begin{aligned}
& \left(\bar{\partial}+\frac{\pi q}{2 \operatorname{Im}(\tau)}(m z+C)\right) \psi_{+}(z, \bar{z})=0 \\
& \left(\partial-\frac{\pi q}{2 \operatorname{Im}(\tau)}(m \bar{z}+\bar{C})\right) \psi_{-}(z, \bar{z})=0
\end{aligned}
$$

where $C_{1}$ and $C_{2}$ are real constants. That is, we can introduce the Wilson line background, $C=C_{1}+\tau C_{2}$ by replacing $\chi_{i}$ in (8) as [7]

$$
\chi_{1}=\frac{\pi}{\operatorname{Im} \tau} \operatorname{Im}(m z+C), \quad \chi_{2}=\frac{\pi}{\operatorname{Im} \tau} \operatorname{Im}(\bar{\tau}(m z+C)) .
$$

Because of this Wilson line, the number of zero-modes does not change, but their wavefunctions are replaced as

$$
\Theta^{j, M}(z) \rightarrow \Theta^{j, M}(z+C / m)
$$

In general, Yukawa couplings are computed by the overlap integral of three zero-mode profiles, $\psi_{i}(z), \psi_{j}(z)$ and $\psi_{k}(z)$,

$$
y_{i j k}=g \int d^{2} z \psi_{i}(z) \psi_{j}(z) \psi_{k}(z),
$$

where $g$ denotes the corresponding coupling in the higher dimensional theory. Concretely, when Wilson lines are vanishing, the overlap integral of $\Theta^{i, M_{1}}(z) \Theta^{j, M_{2}}(z)\left(\Theta^{k, M_{3}}(z)\right)^{*}$ for $M_{3}=M_{1}+M_{2}$ is given $[7,28]$ 4

$$
\begin{aligned}
& \int d^{2} z \Theta^{i, M_{1}}(z) \Theta^{j, M_{2}}(z)\left(\Theta^{k, M_{3}}(z)\right)^{*} \\
& =\frac{N_{M_{1}} N_{M_{2}}}{N_{M_{3}}} \sum_{m \in \mathbf{Z}_{M_{3}}} \delta_{i+j+M_{1} m, k} \times \vartheta\left[\begin{array}{c}
\frac{M_{2} i-M_{1} j+M_{1} M_{2} m}{M_{1} M_{2} M_{3}} \\
0
\end{array}\right]\left(0, \tau M_{1} M_{2} M_{3}\right),
\end{aligned}
$$

where the gauge invariance requires the third wavefuction must be $\left(\Theta^{k, M_{3}}(z)\right)^{*}$ with the magnetic flux $M_{3}=M_{1}+M_{2}$, but not $\Theta^{k, M_{3}}(z)$. Here we have used the product rule (17)

\footnotetext{
${ }^{4}$ See also [29].
} 
and the orthogonality (16). When we introduce non-vanishing Wilson lines, the overlap integral is obtained as

$$
\begin{aligned}
& \int d^{2} z \Theta^{i, M_{1}}\left(z+C / M_{1}\right) \Theta^{j, M_{2}}\left(z+C^{\prime} / M_{2}\right)\left(\Theta^{k, M_{3}}\left(z+C^{\prime \prime} / M_{3}\right)\right)^{*} \\
& \left.=\frac{N_{M_{1}} N_{M_{2}}}{N_{M_{3}}} \sum_{m \in \mathbf{Z}_{M_{3}}} \delta_{i+j+M_{1} m, k} \times \vartheta\left[\begin{array}{c}
\frac{M_{2} i-M_{1} j+M_{1} M_{2} m}{M_{1} M_{2} M_{3}} \\
0
\end{array}\right]\left(M_{2} C-M_{1} C^{\prime}\right), \tau M_{1} M_{2} M_{3}\right),
\end{aligned}
$$

where the gauge invariance requires $C^{\prime \prime}=C+C^{\prime}$. Furthermore, by repeating the above procedure we can compute higher order couplings [30].

In Eqs. (23) and (24), the number of the Kronecker delta is defined modulo $M_{3}$ and the Kronecker delta leads to the selection rule for allowed couplings as

$$
i+j-k=M_{3} \ell-M_{1} m,
$$

where $\ell, m$ are integers. When $\operatorname{gcd}\left(M_{1}, M_{2}, M_{3}\right)=g$, the above constraint becomes

$$
i+j=k, \quad(\bmod g) .
$$

That implies that we can define $Z_{g}$ charge for zero-modes and the allowed couplings are controlled by such a $Z_{g}$ symmetry $[10,30] 5$ This $Z_{g}$ transformation can be written as [10]

$$
Z=\left(\begin{array}{ccccc}
1 & & & & \\
& \rho & & & \\
& \rho^{2} & & \\
& & \ddots & \\
& & & & \rho^{g-1}
\end{array}\right)
$$

where $\rho=e^{2 \pi i / g}$. Furthermore, 4D effective theory has a cyclic permutation symmetry

$$
\Theta^{i, M_{1}} \rightarrow \Theta^{i+m n_{1}, M_{1}}, \quad \Theta^{j, M_{2}} \rightarrow \Theta^{j+m n_{2}, M_{2}}, \quad \Theta^{k, M_{3}} \rightarrow \Theta^{k+m n_{3}, M_{3}},
$$

where $n_{i}=M_{i} / g$ and $m$ is a universal integer, that is, another $Z_{g}$ symmetry. This $Z_{g}$ transformation can be written as [10]

$$
C=\left(\begin{array}{cccccc}
0 & 1 & 0 & 0 & \cdots & 0 \\
0 & 0 & 1 & 0 & \cdots & 0 \\
& & & & \ddots & \\
1 & 0 & 0 & 0 & \cdots & 0
\end{array}\right) .
$$

These two $Z_{g}$ symmetries are non-commutable and lead to non-Abelian flavor symmetry, $\left(Z_{g} \times Z_{g}\right) \rtimes Z_{g}[10]$. Its diagonal elements are written as $Z^{m}\left(Z^{\prime}\right)^{n}$, where

$$
Z^{\prime}=\left(\begin{array}{ccc}
\rho & & \\
& \ddots & \\
& & \rho
\end{array}\right) .
$$

\footnotetext{
${ }^{5}$ See for the same selection rule in intersecting D-brane models [31,32].
} 
These symmetries are also available for higher order couplings. Furthermore, when we consider vanishing Wilson lines, the $Z_{2}$ twist symmetry is enhanced by the symmetry,

$$
\Theta^{i, M} \rightarrow \Theta^{M-i, M}
$$

and such $Z_{2}$ can be written as

$$
\mathcal{P}=\left(\begin{array}{ccccc}
1 & \cdots & \cdots & \cdots & 0 \\
0 & \cdots & \cdots & 0 & 1 \\
0 & \cdots & 0 & 1 & 0 \\
\vdots & & . \cdot & & \vdots \\
0 & 1 & 0 & \cdots & 0
\end{array}\right)
$$

Then, the permutation symmetry is enhanced from $Z_{g}$ to $D_{g}$, and the total symmetry becomes $\left(Z_{g} \times Z_{g}\right) \rtimes D_{g}$. For example, when $g=3$, we can realize $\left(Z_{3} \times Z_{3}\right) \rtimes Z_{3}=\Delta(27)$ and $\left(Z_{3} \times Z_{3}\right) \rtimes D_{3}=\Delta(54)$.

It would be useful to consider $U(1)_{a} \times U(1)_{b}$ theory from the phenomenological viewpoint. We consider the fermion field $\lambda(x, z)$ with $U(1)_{a} \times U(1)_{b}$ charges, $\left(q_{a}, q_{b}\right)$. We assume the following form of $U(1)_{a}$ magnetic flux on $T^{2}$,

$$
F_{z \bar{z}}^{a}=\frac{\pi i}{\operatorname{Im} \tau} m_{a}
$$

where $m_{a}$ is integer, but there is no magnetic flux in $U(1)_{b}$. On top of that, we introduce Wilson lines $C^{a}$ and $C^{b}$ for $U(1)_{a}$ and $U(1)_{b}$, respectively. The zero-mode equations are written as

$$
\begin{aligned}
& \left(\bar{\partial}+\frac{\pi}{2 \operatorname{Im}(\tau)}\left(q_{a}\left(m_{a} z+C^{a}\right)+q_{b} C^{b}\right)\right) \psi_{+}(z, \bar{z})=0 \\
& \left(\partial-\frac{\pi}{2 \operatorname{Im}(\tau)}\left(q_{a}\left(m_{a} \bar{z}+\bar{C}^{a}\right)+q_{b} \bar{C}^{b}\right)\right) \psi_{-}(z, \bar{z})=0 .
\end{aligned}
$$

Then, the number of zero-modes is obtained as $M=q_{a} m_{a}$ and their wavefunctions are written as

$$
\Theta^{j, M}\left(z+C / m_{a}\right)
$$

where $C=C^{a}+C^{b} q_{b} / q_{a}$. Here we give a few comments. All of modes with $q_{a}=0$ become massive and there do not appear zero-modes with $q_{a}=0$. For $q_{a} \neq 0$, zeromodes with $q_{b}=0$ appear and the number of zero-modes is independent of $q_{b}$. Obviously, when we introduce Wilson lines $C^{a}$ and/or $C^{b}$ without magnetic flux $F^{a}$, zero-modes do not appear. The shift of wavefunctions depends on $1 / m_{a}$ and the charge $q_{b}$. Note that although $F^{b}=0$, Wilson lines $C_{b}$ and charges $q_{b}$ for $U(1)_{b}$ are also important 6 .

\footnotetext{
${ }^{6}$ Wilson lines $C_{b}$ and charges $q_{b}$ for $U(1)_{b}$ are in a sense more important than Wilson lines $C_{a}$ and charges $q_{a}$ for $U(1)_{a}$, because the shift of wavefunctions (36) depends on $q_{b}$.
} 


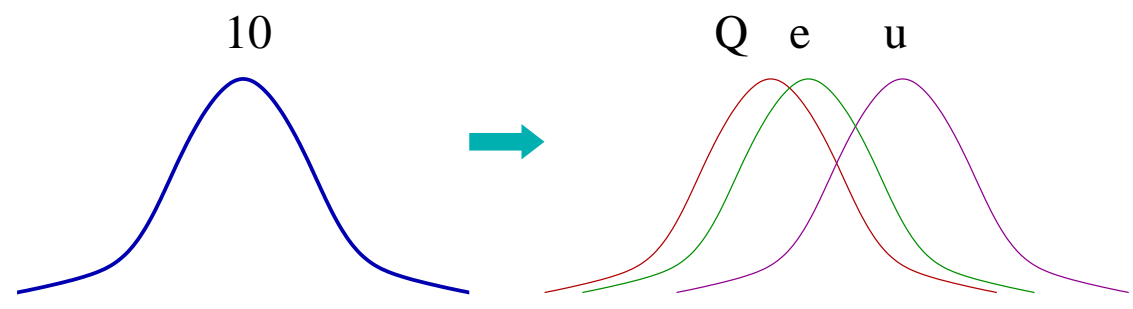

Figure 1: Wavefunction splitting by Wilson lines

The above aspects of magnetic fluxes and Wilson lines are phenomenologically interesting. We consider 6D super Yang-Mills theory with non-Abelian gauge group $G$. We introduce a magnetic flux $F^{a}$ along a Cartan direction of $G$. Then, the gauge group breaks to $G^{\prime} \times U(1)_{a}$ without reducing the rank. Furthermore, there appear the massless fermion fields $\lambda^{\prime}$, which correspond to the gaugino fields for the broken gauge group part and have the fundamental representation of $G^{\prime}$ and non-vanishing $U(1)_{a}$ charge. Furthermore, we introduce Wilson line along a Cartan direction of $G^{\prime}$. Then, the gauge group is broken to $G^{\prime \prime} \times U(1)_{a} \times U(1)_{b}$ without reducing the rank. The gaugino fields corresponding to the broken gauge part in $G^{\prime}$ do not remain as massless modes, but they gain masses due to the Wilson line $U(1)_{b}$. However, the fermion fields $\lambda^{\prime}$ remain still massless with the same degeneracy.

Let us explain more on this aspect. Suppose that we introduce magnetic fluxes in a model with a larger group $G$ such that they break $G$ to a GUT group like $S U(5)$ and this model include three families of matter fields like 10 and $\overline{5}$. Their Yukawa couplings are computed by the overlap integral of three zero-mode profiles as Eq. (22). We obtain the GUT relation among Yukawa coupling matrices when wavefunction profiles of matter fields in $10(\overline{5})$ are degenerate like Eq. (23) . Then, we introduce a Wilson line along $U(1)_{Y}$, which breaks $S U(5)$ to $S U(3) \times S U(2) \times U(1)_{Y}$. Because of Wilson lines, $S U(5)$ gauge bosons except the $S U(3) \times S U(2) \times U(1)_{Y}$ gauge bosons become massive and the corresponding gaugino fields become massive. However, three families of 10 and $\overline{5}$ matter fields remain massless. Importantly, this Wilson line resolves the degeneracy of wavefunction profiles of left-handed quarks, right-handed up-sector quarks and right-handed charged leptons in 10 and right-handed down-sector quarks and left-handed charged leptons in $\overline{5}$ as Figure 1. That is, the GUT relation among Yukawa coupling matrices is deformed. As an illustrating model, we study the Pati-Salam model in the next subsection.

Here we study effects due to discrete values of Wilson lines such as $C=k \tau$ with $k=$ integer. We find

$$
\Theta^{j, M}(z+k \tau / M)=e^{\pi i k \operatorname{Im}(\bar{\tau} z) / \operatorname{Im}(\tau)} \Theta^{j+k, M}(z) .
$$

Thus, the effect of discrete Wilson lines $C=k \tau$ is to replace the $j$-th zero-mode by the $(j+k)$-th zero-mode up to $e^{\pi i k \operatorname{Im}(\bar{\tau} z) / \operatorname{Im}(\tau)}$. However, when we consider 3-point and higher order couplings, the gauge invariance requires that the sum of Wilson lines of matter fields should vanish, that is, $\sum_{i} k_{i}=0$ for allowed n-point couplings. Thus, the part 
$e^{\pi i k \operatorname{Im}(\bar{\tau} z) / \operatorname{Im}(\tau)}$ is irrelevant to $4 \mathrm{D}$ effective theory and the resultant $4 \mathrm{D}$ effective theory is the equivalent even when we introduce $C=k \tau$. Similarly, introducing the Wilson lines $C=k$ with $k=$ integer leads to the equivalent $4 \mathrm{D}$ effective theory.

\section{$2.2 \quad$ Pati-Salam model}

As an illustrating model, we consider the Pati-Salam model. We start with 10D N=1 $U(8)$ super Yang-Mills theory with the Lagrangian

$$
\mathcal{L}=-\frac{1}{4 g^{2}} \operatorname{Tr}\left(F^{M N} F_{M N}\right)+\frac{i}{2 g^{2}} \operatorname{Tr}\left(\bar{\lambda} \Gamma^{M} D_{M} \lambda\right),
$$

where $M, N=0, \cdots, 9$. We compactify the extra 6 dimensions on $T_{1}^{2} \times T_{2}^{2} \times T_{3}^{2}$, and we denote the complex coordinate for the $d$-th $T_{d}^{2}$ by $z^{d}$, where $d=1,2,3$. Then, we introduce the following form of magnetic fluxes,

$$
F_{z^{d} \bar{z}^{d}}=\frac{\pi i}{\operatorname{Im} \tau_{d}}\left(\begin{array}{ccc}
m_{1}^{(d)} \mathbb{1}_{4} & & \\
& m_{2}^{(d)} \mathbb{1}_{2} & \\
& & m_{3}^{(d)} \mathbb{1}_{2}
\end{array}\right), \quad d=1,2,3,
$$

in the gauge space, where $\mathbb{1}_{N}$ are the unit matrices of rank $N, m_{i}^{(d)}$ are integers. We assume that the above background preserves $4 \mathrm{D} \mathrm{N}=1$ supersymmetry (SUSY). Here, we denote $M_{i j}^{(d)}=m_{i}^{(d)}-m_{j}^{(d)}$ and $M_{i j}=M_{i j}^{(1)} M_{i j}^{(2)} M_{i j}^{(3)}$. This magnetic flux breaks the gauge group $U(8)$ to $U(4) \times U(2)_{L} \times U(2)_{R}$, that is the Pati-Salam gauge group up to $U(1)$ factors. The gauge sector corresponds to $4 \mathrm{D} N=4$ SUSY vector multiplet, that is, there are $U(4) \times U(2)_{L} \times U(2)_{R} \mathrm{~N}=1$ vector multiplet and three adjoint chiral multiplets. In addition, there appear bifundamental matter fields like $\lambda_{(4,2,1)}, \lambda_{(\overline{4}, 1,2)}$ and $\lambda_{(1,2,2)}$, and their numbers of zero-modes are equal to $M_{12}, M_{31}$ and $M_{23}$. When $M_{i j}$ is negative, that implies their conjugate matter fields appear with the degeneracy $\left|M_{i j}\right|$. The fields $\lambda_{(4,2,1)}$ and $\lambda_{(\overline{4}, 1,2)}$ correspond to left-handed and right-handed matter fields, respectively, while $\lambda_{(1,2,2)}$ corresponds to up and down Higgs (higgsino) fields. For example, we can realize three families by $M_{12}^{(d)}=(3,1,1)$ and $M_{31}^{(d)}=(3,1,1)$. That leads to $\left|M_{23}\right|=0$ or 24 . At any rate, the flavor structure is determined by the first $T_{1}^{2}$ in such a model. Explicitly, the zero-mode wavefunctions of both $\lambda_{(4,2,1)}$ and $\lambda_{(\overline{4}, 1,2)}$ are obtained as

$$
\Theta^{j, 3}\left(z^{1}\right) \Theta^{1,1}\left(z^{2}\right) \Theta^{1,1}\left(z^{3}\right) .
$$

Their Yukawa matrices are constrained by the Pati-Salam gauge symmetry, that is, up-sector quarks, down-sector quarks, charged leptons and neutrinos have the same Yukawa matrices with Higgs fields. Even with such a constraint, one could derive realistic quark/lepton masses and mixing angles, because this model has many Higgs fields and their vacuum expectation values generically break the up-down symmetry.

We introduce Wilson lines in $U(4)$ and $U(2)_{R}$ such that $U(4)$ breaks to $U(1) \times U(3)$ and $U(2)_{R}$ breaks $U(1) \times U(1)$. Then, the gauge group becomes the standard gauge group up 
to $U(1)$ factors. Furthermore, the profiles of left-handed quarks and leptons in $\lambda_{(4,2,1)}$ shift differently because of Wilson lines. Similarly, right-handed up-sector quarks, down-sector quarks, charged leptons and neutrinos in $\lambda_{(\overline{4}, 1,2)}$ shift differently. The flavor structure is determined by the first $T_{1}^{2}$. Thus, when we introduce Wilson lines the second or third torus, the resultant Yukawa matrices are constrained by the $S U(4) \times S U(2)_{L} \times S U(2)_{R}$. For example, we introduce Wilson lines on $T_{2}^{2}$. Then, zero-mode profiles of quarks, $(Q, u, d)$ and leptons $(L, e, \nu)$ split as

$$
\begin{aligned}
Q: & \Theta^{j, 3}\left(z^{1}\right) \Theta^{1,1}\left(z^{2}+C^{a}\right) \Theta^{1,1}\left(z^{3}\right), \\
L: & \Theta^{j, 3}\left(z^{1}\right) \Theta^{1,1}\left(z^{2}-3 C^{a}\right) \Theta^{1,1}\left(z^{3}\right), \\
u^{c}: & \Theta^{j, 3}\left(z^{1}\right) \Theta^{1,1}\left(z^{2}-C^{a}+C^{b}\right) \Theta^{1,1}\left(z^{3}\right), \\
d^{c}: & \Theta^{j, 3}\left(z^{1}\right) \Theta^{1,1}\left(z^{2}-C^{a}-C^{b}\right) \Theta^{1,1}\left(z^{3}\right), \\
e^{c}: & \Theta^{j, 3}\left(z^{1}\right) \Theta^{1,1}\left(z^{2}+3 C^{a}-C^{b}\right) \Theta^{1,1}\left(z^{3}\right), \\
\nu^{c}: & \Theta^{j, 3}\left(z^{1}\right) \Theta^{1,1}\left(z^{2}+3 C^{a}+C^{b}\right) \Theta^{1,1}\left(z^{3}\right),
\end{aligned}
$$

where $C^{a}$ and $C^{b}$ are the Wilson lines to break $U(4) \rightarrow U(3) \times U(1)$ and $U(2)_{R} \rightarrow$ $U(1) \times U(1)$, respectively. Those Wilson lines just change the overall factors of Yukawa matrices, but ratios among elements in one Yukawa matrix do not change. Also we can introduce Wilson lines along the same $U(1)$ directions as the magnetic fluxes (39), but they do not deform the up-down symmetry of Yukawa matrices, either.

On the other hand, when we introduce Wilson lines on the first $T_{1}^{2}$, the zero-mode wavefunctions split as

$$
\begin{aligned}
& Q: \Theta^{j, 3}\left(z^{1}+C^{a} / 3\right) \Theta^{1,1}\left(z^{2}\right) \Theta^{1,1}\left(z^{3}\right), \\
& L: \Theta^{j, 3}\left(z^{1}-C^{a}\right) \Theta^{1,1}\left(z^{2}\right) \Theta^{1,1}\left(z^{3}\right) \text {, } \\
& u^{c}: \Theta^{j, 3}\left(z^{1}-C^{a} / 3+C^{b} / 3\right) \Theta^{1,1}\left(z^{2}\right) \Theta^{1,1}\left(z^{3}\right) \text {, } \\
& d^{c}: \Theta^{j, 3}\left(z^{1}-C^{a} / 3-C^{b} / 3\right) \Theta^{1,1}\left(z^{2}\right) \Theta^{1,1}\left(z^{3}\right) \text {, } \\
& e^{c}: \Theta^{j, 3}\left(z^{1}+C^{a}-C^{b} / 3\right) \Theta^{1,1}\left(z^{2}\right) \Theta^{1,1}\left(z^{3}\right) \text {, } \\
& \nu^{c}: \Theta^{j, 3}\left(z^{1}+C^{a}+C^{b} / 3\right) \Theta^{1,1}\left(z^{2}\right) \Theta^{1,1}\left(z^{3}\right) \text {. }
\end{aligned}
$$

In this case, the flavor structure is deviated from the $S U(4) \times S U(2)_{L} \times S U(2)_{R}$ relation, that is, mass ratios and mixing angles can change. Also we can introduce Wilson lines $C^{a}$ to $T_{2}^{2}$ and $C^{b}$ to $T_{1}^{2}$. Then we realize

$$
\begin{aligned}
& Q: \Theta^{j, 3}\left(z^{1}\right) \Theta^{1,1}\left(z^{2}+C^{a}\right) \Theta^{1,1}\left(z^{3}\right), \\
& L: \Theta^{j, 3}\left(z^{1}\right) \Theta^{1,1}\left(z^{2}-3 C^{a}\right) \Theta^{1,1}\left(z^{3}\right) \text {, } \\
& u^{c}: \Theta^{j, 3}\left(z^{1}+C^{b} / 3\right) \Theta^{1,1}\left(z^{2}-C^{a}\right) \Theta^{1,1}\left(z^{3}\right) \text {, } \\
& d^{c}: \Theta^{j, 3}\left(z^{1}-C^{b} / 3\right) \Theta^{1,1}\left(z^{2}-C^{a}\right) \Theta^{1,1}\left(z^{3}\right) \text {, } \\
& e^{c}: \Theta^{j, 3}\left(z^{1}-C^{b} / 3\right) \Theta^{1,1}\left(z^{2}+3 C^{a}\right) \Theta^{1,1}\left(z^{3}\right) \text {, } \\
& \nu^{c}: \Theta^{j, 3}\left(z^{1}+C^{b} / 3\right) \Theta^{1,1}\left(z^{2}+3 C^{a}\right) \Theta^{1,1}\left(z^{3}\right) \text {. }
\end{aligned}
$$

Indeed, this behavior is well-known in the intersecting D-brane models, which are Tduals of magnetized D-brane models. In the intersecting D-brane side, introduction of 


\begin{tabular}{|c||c|c|}
\hline & $M=$ even & $M=$ odd \\
\hline \hline even zero-modes & $M / 2+1$ & $(M+1) / 2$ \\
\hline odd zero-modes & $M / 2-1$ & $(M-1) / 2$ \\
\hline
\end{tabular}

Table 1: The numbers of zero-modes for even and odd wavefunctions.

Wilson lines corresponds to split D-branes. By splitting D-branes, the gauge group breaks as $U(M+N) \rightarrow U(M) \times U(N)$, but the number of massless bi-fundamental modes does not change, although they decompose because of the gauge symmetry breaking.

\section{Orbifold models}

Here, we study orbifold models with magnetic fluxes and Wilson lines. The $T^{2} / Z_{2}$ orbifold is constructed by identifying $z \sim-z$ on $T^{2}$. We also embed the $Z_{2}$ twist into the gauge space as $P$. Note that under the $Z_{2}$ twist, magnetic flux background is invariant. That is, we have no constraint on magnetic fluxes due to orbifolding. Furthermore, zero-mode wavefunctions satisfy

$$
\Theta^{j, M}(-z)=\Theta^{M-j, M}(z)
$$

Note that $\Theta^{0, M}(z)=\Theta^{M, M}(z)$. Hence, the $Z_{2}$ eigenstates are written as [19]

$$
\Theta_{ \pm}^{j, M}(z)=\frac{1}{\sqrt{2}}\left(\Theta^{j, M}(z) \pm \Theta^{M-j, M}(z)\right)
$$

for $j \neq 0, M / 2, M$. The wavefunctions $\Theta^{j, M}(z)$ for $j=0, M / 2$ are the $Z_{2}$ eigenstates with the $Z_{2}$ even parity. Either of $\Theta_{+}^{j, M}(z)$ and $\Theta_{-}^{j, M}(z)$ is projected out by the orbifold projection. Odd wavefunctions can also correspond to massless modes in the magnetic flux background, although on the orbifold without magnetic flux odd modes always correspond to massive modes, but not massless modes. Before orbifolding, the number of zero-modes is equal to the magnetic flux $M$. For example, we have to choose $M=3$ in order to realize the three families. On the other hand, the number of zero-modes on the orbifold also depends on the boundary conditions under the $Z_{2}$ twist, even or odd functions. For $M=$ even, the number of zero-modes with even (odd) functions are equal to $M / 2+1$ $(M / 2-1)$. For $M=$ odd, the number of zero-modes with even and odd functions are equal to $(M+1) / 2$ and $(M-1) / 2$, respectively. These results are shown in Table 1 . For example, when we choose even (odd) functions, the three families can be realized for $M=4$ and 5 ( 7 and 8 ). Thus, we can obtain various three-family models in magnetized orbifold models and those have richer flavor structure than torus models with magnetic fluxes. Yukawa couplings among $\Theta_{ \pm}^{i, M_{1}}(z) \Theta_{ \pm}^{j, M_{2}}(z)\left(\Theta_{ \pm}^{k, M_{3}}(z)\right)^{*}$ are computed by use of Eq. (23).

Now, let us introduce Wilson lines [33]. We consider $U(1)_{a} \times S U(2)$ theory. Then we introduce magnetic flux in $U(1)_{a}$ like Eq. (33). In addition, we embed the $Z_{2}$ twist $P$ into 
the $S U(2)$ gauge space. For example, we consider the $S U(2)$ doublet

$$
\left(\begin{array}{c}
\lambda_{1 / 2} \\
\lambda_{-1 / 2}
\end{array}\right),
$$

with the $U(1)_{a}$ charge $q_{a}$. We embed the $Z_{2}$ twist $P$ in the gauge space as

$$
P=\left(\begin{array}{ll}
0 & 1 \\
1 & 0
\end{array}\right)
$$

for the doublet. Obviously, we can diagonalize $P$ as $P^{\prime}=\operatorname{diag}(1,-1)$, if there is no Wilson line along the other $S U(2)$ directions. However, we introduce a Wilson line along the Cartan direction of $S U(2)$, i.e, the following direction

$$
\left(\begin{array}{cc}
1 & 0 \\
0 & -1
\end{array}\right)
$$

in the $P$ basis. Thus, we use the above basis for $P$. For the $S U(2)$ gauge sector, there is no effect due to the magnetic flux. Then, the situation is the same as one on the orbifold without magnetic flux. The $S U(2)$ gauge group is broken completely, that is, all of $S U(2)$ vector multiplets become massive.

Before orbifolding, the $S U(2)$ is not broken and both $\lambda_{1 / 2}$ and $\lambda_{-1 / 2}$ have $M=q_{a} m_{a}$ independent zero-modes, which we denote by $\Theta_{1 / 2}^{j, M}(z)$ and $\Theta_{-1 / 2}^{j, M}(z)$, respectively. Here, we have put the indices, $1 / 2$ and $-1 / 2$ in order to make it clear that they correspond to $\lambda_{1 / 2}$ and $\lambda_{-1 / 2}$, respectively. However, the form of wavefunctions are the same, i.e. $\Theta_{1 / 2}^{j, M}(z)=\Theta_{-1 / 2}^{j, M}(z)$. When we impose the orbifold boundary conditions with the above $P$ in (47), the zero-modes on the orbifold without Wilson lines are written as

$$
\frac{1}{\sqrt{2}}\left(\Theta_{1 / 2}^{j, M}(z)+\Theta_{-1 / 2}^{M-j, M}(z)\right)
$$

for $j=0, \cdots, M-1$. Note that there are $M$ independent zero-modes. It may be useful to explain remaining zero-modes in the basis for $P^{\prime}$. Before orbifolding, both $\lambda_{1 / 2}^{\prime}$ and $\lambda_{-1 / 2}^{\prime}$ have $M=q_{a} m_{a}$ independent zero-modes in the basis for $P^{\prime}$. Then by orbifolding with $P^{\prime}$, even modes corresponding to $\Theta_{+}^{j, M}(z)$ remain for $\lambda_{1 / 2}^{\prime}$, while $\lambda_{-1 / 2}^{\prime}$ has only odd modes $\Theta_{-}^{j, M}(z)$. Their total number is equal to $M$.

Then, we introduce the Wilson lines along the Cartan direction in the basis for $P$. The corresponding zero-mode wavefunctions are shifted as

$$
\frac{1}{\sqrt{2}}\left(\Theta_{1 / 2}^{j, M}\left(z+C^{b} / 2 M\right)+\Theta_{-1 / 2}^{M-j, M}\left(z-C^{b} / 2 M\right)\right)
$$

for $j=0, \cdots, M-1$, where $C^{b}$ is a continuous parameter. Note that $\lambda_{1 / 2}$ and $\lambda_{-1 / 2}$ have opposite charges under the $S U(2)$ Cartan. Then, their wavefunctions are shifted to opposite directions by the same Wilson lines $C^{b}$ as $\Theta_{1 / 2}^{j, M}\left(z+C^{b} / 2 M\right)$ and $\Theta_{-1 / 2}^{j, M}(z-$ 
$\left.C^{b} / 2 M\right)$. We can also consider the $Z_{2}$ twist $P$ in the doublet such that the following wavefunction

$$
\frac{1}{\sqrt{2}}\left(\Theta_{1 / 2}^{j, M}\left(z+C^{b} / 2 M\right)-\Theta_{-1 / 2}^{M-j, M}\left(z-C^{b} / 2 M\right)\right)
$$

remains.

For comparison, we study another dimensional representations, e.g. a triplet

$$
\left(\begin{array}{c}
\lambda_{1} \\
\lambda_{0} \\
\lambda_{-1}
\end{array}\right)
$$

with the $U(1)_{a}$ charge $q_{a}$. Suppose that we embed the $Z_{2}$ twist $P$ in the three dimensional gauge space as

$$
P=\left(\begin{array}{lll}
0 & 0 & 1 \\
0 & 1 & 0 \\
1 & 0 & 0
\end{array}\right)
$$

for the triplet. Then, zero-modes on the orbifold are written as

$$
\begin{aligned}
& \Theta_{1}^{j, M}(z)+\Theta_{-1}^{M-j, M}(z), \\
& \Theta_{0}^{j, M}(z)+\Theta_{0}^{M-j, M}(z),
\end{aligned}
$$

up to the normalization factor $1 / \sqrt{2}$. The former corresponds to $\lambda_{1}$ and $\lambda_{-1}$ and there are $M$ zero-modes. The latter corresponds to $\lambda_{0}$ and there are $(M / 2+1)$ zero-modes and $(M+1) / 2$ zero-modes when $M$ is even and odd, respectively. When we introduce the continuous Wilson lines along the Cartan direction, these zero-modes shift as

$$
\begin{aligned}
& \Theta_{1}^{j, M}\left(z+C_{b} / M\right)+\Theta_{-1}^{M-j, M}\left(z-C_{b} / M\right), \\
& \Theta_{0}^{j, M}(z)+\Theta_{0}^{M-j, M}(z) .
\end{aligned}
$$

We can extend the above analysis to larger gauge groups. Here, we show a rather simple example. We consider $U(1)_{a} \times S U(3)$ theory with the magnetic flux in $U(1)_{a}$ like Eq. (33). Then, we consider the $S U(3)$ triplet,

$$
\left(\begin{array}{c}
\lambda_{0} \\
\lambda_{1 / 2} \\
\lambda_{-1 / 2}
\end{array}\right)
$$

with the $U(1)_{a}$ charge $q_{a}$, where the subscripts $(0,1 / 2,-1 / 2)$ denote the $U(1)_{b}$ charge along one of $S U(3)$ Cartan directions. Now, we embed the $Z_{2}$ twist $P$ in the gauge space as

$$
P=\left(\begin{array}{lll}
1 & 0 & 0 \\
0 & 0 & 1 \\
0 & 1 & 0
\end{array}\right)
$$


for the triplet. In addition, we introduce the Wilson line $C^{b}$ along the $U(1)_{b}$ direction. The gauge group is broken as $S U(3) \rightarrow U(1) 7$ There are $M$ zero-modes for linear combinations of $\lambda_{1 / 2}$ and $\lambda_{-1 / 2}$ with the wavefunctions,

$$
\Theta_{1 / 2}^{j, M}\left(z+C^{b} / 2 M\right)+\Theta_{-1 / 2}^{M-j, M}\left(z-C^{b} / 2 M\right),
$$

up to the normalization factor. Also, the zero-modes for $\lambda_{0}$ are written as

$$
\Theta_{0}^{j, M}(z)+\Theta_{0}^{M-j, M}(z)
$$

up to the normalization factor. The number of zero-modes is equal to $(M / 2+1)$ and $(M+1) / 2$ when $M$ is even and odd, respectively. Thus, the situation is almost the same as the above $S U(2)$ case with the triplet. Although the above example is rather simple, we can consider various types of breaking for larger groups. For example, when the gauge group includes two or more $S U(2)$ subgroups, we could embed the $Z_{2}$ twist in two of $S U(2)$ 's and introduce independent Wilson lines along their Cartan directions. Similarly, we can investigate such models and other types of various embedding of $P$ and Wilson lines.

In section 2.2 , we have considered $10 \mathrm{D}$ theory on $T^{6}$. Also, we can consider the $T^{6} / Z_{2}$ orbifold, where the $Z_{2}$ twist acts e.g.

$$
Z_{2}: z_{1} \rightarrow-z_{1}, \quad z_{2} \rightarrow-z_{2}, \quad z_{3} \rightarrow z_{3}
$$

For $T_{1}^{2}$ and $T_{2}^{2}$, we can introduce the type of Wilson lines, which we have considered in this section, while for $T_{3}^{2}$ we can introduce the type of Wilson lines, which are considered in the previous section. Then, we have a richer structure of models on the $T^{6} / Z_{2}$ orbifold. Furthermore, we could consider another independent $Z_{2}^{\prime}$ twist as

$$
Z_{2}: z_{1} \rightarrow-z_{1}, \quad z_{2} \rightarrow z_{2}, \quad z_{3} \rightarrow-z_{3},
$$

on the $T^{6} /\left(Z_{2} \times Z_{2}^{\prime}\right)$ orbifold. In this case, we can consider another independent embedding $P^{\prime}$ of $Z_{2}^{\prime}$ twist on the gauge space. Using these two $Z_{2}$ twist embedding and Wilson lines, we could construct various types of models. For example, when the gauge group includes two or more $S U(2)$ subgroups, we could embed $P$ on one of $S U(2)$ and $P^{\prime}$ on other $S U(2)$ and introduce independent Wilson lines along their Cartan directions. Other various types of model building would be possible. Thus, it would be interesting to study such model building elsewhere.

Finally, we comment on the flavor symmetry. Yukawa couplings as well as higher order couplings can be computed by use of Eq. (24). The orbifolding without Wilson lines is a procedure to choose eigenstates for $\mathcal{P}(32)$. Thus, there remains the flavor symmetry, which commutes with $\mathcal{P}$. The $Z_{g}^{\prime}$ symmetry (30) is commutable. The $Z_{g}$ symmetry (27) is not commutable for $g=$ odd. However, when $g=$ even, the $Z_{2}$ symmetry, which is

7 This remaining $U(1)$ symmetry might be anomalous. If so, the remaining $U(1)$ would also be broken by the Green-Schwarz mechanism. 
generated by $Z^{g / 2}$ is commutable with $\mathcal{P}$ and another $Z_{2}$ symmetry, which is generated by $C^{g / 2}$, is also commutable with $\mathcal{P}$. For example, when $g=4$, the generators, $Z^{\prime}, Z^{2}$ and $C^{2}$ are written as

$$
Z^{\prime}=\left(\begin{array}{ccc}
i & & \\
& \ddots & \\
& & i
\end{array}\right), \quad Z^{2}=\left(\begin{array}{cccc}
1 & & & \\
& -1 & & \\
& & 1 & \\
& & & -1
\end{array}\right), \quad C^{2}=\left(\begin{array}{llll}
0 & 0 & 1 & 0 \\
0 & 0 & 0 & 1 \\
1 & 0 & 0 & 0 \\
0 & 1 & 0 & 0
\end{array}\right) .
$$

Here, the $Z^{2}$ and $C^{2}$ generators also commute with each other. Similarly, when $g / 2=$ even, the unbroken flavor symmetry would be obtained as $Z_{g} \times Z_{2} \times Z_{2} \times Z_{2} 8$ On the other hand, when $g / 2=3$, the generators, $Z^{\prime}, Z^{3}$ and $C^{3}$ are written as

$$
\begin{aligned}
& Z^{\prime}=\left(\begin{array}{ccc}
\rho & & \\
& \ddots & \\
& & \rho
\end{array}\right), \\
& Z^{3}=\left(\begin{array}{cccccc}
1 & & & & & \\
& -1 & & & & \\
& & 1 & & & \\
& & & -1 & & \\
& & & & 1 & \\
& & & & & -1
\end{array}\right), \quad C^{3}=\left(\begin{array}{llllll}
0 & 0 & 0 & 1 & 0 & 0 \\
0 & 0 & 0 & 0 & 1 & 0 \\
0 & 0 & 0 & 0 & 0 & 1 \\
1 & 0 & 0 & 0 & 0 & 0 \\
0 & 1 & 0 & 0 & 0 & 0 \\
0 & 0 & 1 & 0 & 0 & 0
\end{array}\right)
\end{aligned}
$$

where $\rho=e^{\pi i / 3}$. Here, the $Z^{3}$ and $C^{3}$ generators do not commute with each other. Thus, unbroken flavor symmetries are non-Abelian. Similarly, when $g / 2=$ odd, non-Abelian discrete flavor symmetries would remain.

When we introduce the above Wilson lines, the $S U(2)$ gauge symmetry is broken at the same time as the $Z_{g}$ symmetry breaking for (27). Thus, we may expect that some non-trivial linear combinations of broken $Z_{g}$ and $S U(2)$ would remain. However, only the $Z_{2}$ symmetry, which is already included above, seems to remain e.g. in the states (49). When we consider more complicated models, a new type of flavor symmetries, which are linear combinations of broken flavor symmetries and gauge symmetries, may remain. Hence it would be interesting to investigate such models.

\section{Conclusion and discussion}

We have studied torus/orbifold models with magnetic fluxes and Wilson lines. These backgrounds lead to various different aspects like the number of zero-modes, their profiles, breaking patterns of flavor symmetries, etc. Using these backgrounds, it would be quite interesting to construct concrete models. We would study them elsewhere.

In addition to continuous Wilson lines, we can introduce discrete Wilson lines on the orbifold without magnetic fluxes, which break the gauge group without reducing its rank.

\footnotetext{
${ }^{8}$ It is interesting to break non-Abelian flavor symmetries to Abelian symmetries by orbifolding [34].
} 
It is quite important to study the possibility for introducing discrete Wilson lines in the magnetic background and study their phenomenological implications. Furthermore, it is also important to analyze (systematically) which types of backgrounds and boundary conditions are possible in generic case.

\section{Acknowledgement}

The authors would like to thank R. Maruyama, M. Murata, Y. Nakai and M. Sakai for useful discussions. H. A. is supported in part by the Waseda University Grant for Special Research projects No. 2009A-854. K.-S. C., T. K. and H. O. are supported in part by the Grant-in-Aid for Scientific Research No. 20·08326, No. 20540266 and No. 21.897 from the Ministry of Education, Culture, Sports, Science and Technology of Japan. T. K. is also supported in part by the Grant-in-Aid for the Global COE Program "The Next Generation of Physics, Spun from Universality and Emergence" from the Ministry of Education, Culture,Sports, Science and Technology of Japan.

\section{References}

[1] N. S. Manton, Nucl. Phys. B 193, 502 (1981); G. Chapline and R. Slansky, Nucl. Phys. B 209, 461 (1982); S. Randjbar-Daemi, A. Salam and J. A. Strathdee, Nucl. Phys. B 214, 491 (1983); C. Wetterich, Nucl. Phys. B 222, 20 (1983); P. H. Frampton and K. Yamamoto, Phys. Rev. Lett. 52, 2016 (1984); P. H. Frampton and T. W. Kephart, Phys. Rev. Lett. 53, 867 (1984); K. Pilch and A. N. Schellekens, Nucl. Phys. B 256, 109 (1985);

[2] E. Witten, Phys. Lett. B 149, 351 (1984).

[3] C. Bachas, arXiv:hep-th/9503030.

[4] M. Berkooz, M. R. Douglas and R. G. Leigh, Nucl. Phys. B 480, 265 (1996) arXiv:hep-th/9606139.

[5] R. Blumenhagen, L. Goerlich, B. Kors and D. Lust, JHEP 0010, 006 (2000) arXiv:hep-th/0007024.

[6] C. Angelantonj, I. Antoniadis, E. Dudas and A. Sagnotti, Phys. Lett. B 489, 223 (2000) arXiv:hep-th/0007090.

[7] D. Cremades, L. E. Ibanez and F. Marchesano, JHEP 0405, 079 (2004) arXiv:hep-th/0404229].

[8] J. Alfaro, A. Broncano, M. B. Gavela, S. Rigolin and M. Salvatori, JHEP 0701 (2007) 005 arXiv:hep-ph/0606070.

[9] J. Troost, Nucl. Phys. B 568, 180 (2000) arXiv:hep-th/9909187]. 
[10] H. Abe, K. S. Choi, T. Kobayashi and H. Ohki, Nucl. Phys. B 820, 317 (2009) arXiv:0904.2631 [hep-ph]].

[11] T. Kobayashi, S. Raby and R. J. Zhang, Nucl. Phys. B 704, 3 (2005) [arXiv:hep-ph/0409098].

[12] T. Kobayashi, H. P. Nilles, F. Ploger, S. Raby and M. Ratz, Nucl. Phys. B 768, 135 (2007) arXiv:hep-ph/0611020].

[13] P. Ko, T. Kobayashi, J. h. Park and S. Raby, Phys. Rev. D 76, 035005 (2007) [Erratum-ibid. D 76, 059901 (2007)] [arXiv:0704.2807 [hep-ph]].

[14] I. Antoniadis and T. Maillard, Nucl. Phys. B 716, 3 (2005) arXiv:hep-th/0412008.

[15] G. Aldazabal, S. Franco, L. E. Ibanez, R. Rabadan and A. M. Uranga, J. Math. Phys. 42, 3103 (2001) arXiv:hep-th/0011073]; JHEP 0102, 047 (2001) arXiv:hep-ph/0011132.

[16] R. Blumenhagen, B. Kors and D. Lust, JHEP 0102, 030 (2001) arXiv:hep-th/0012156.

[17] M. Cvetic, G. Shiu and A. M. Uranga, Phys. Rev. Lett. 87, 201801 (2001) arXiv:hep-th/0107143; Nucl. Phys. B 615, 3 (2001) arXiv:hep-th/0107166;

[18] R. Blumenhagen, M. Cvetic, P. Langacker and G. Shiu, Ann. Rev. Nucl. Part. Sci. 55, 71 (2005) arXiv:hep-th/0502005]; R. Blumenhagen, B. Kors, D. Lust and S. Stieberger, Phys. Rept. 445, 1 (2007) [arXiv:hep-th/0610327].

[19] H. Abe, T. Kobayashi and H. Ohki, JHEP 0809, 043 (2008) [arXiv:0806.4748 [hepth]].

[20] H. Abe, K. S. Choi, T. Kobayashi and H. Ohki, Nucl. Phys. B 814, 265 (2009) arXiv:0812.3534 [hep-th]].

[21] J. P. Conlon, A. Maharana and F. Quevedo, JHEP 0809, 104 (2008) arXiv:0807.0789 [hep-th]].

[22] F. Marchesano, P. McGuirk and G. Shiu, arXiv:0812.2247 [hep-th].

[23] P. G. Camara and F. Marchesano, arXiv:0906.3033 [hep-th].

[24] R. Blumenhagen, M. Cvetic, F. Marchesano and G. Shiu, JHEP 0503, 050 (2005) arXiv:hep-th/0502095.

[25] G. 't Hooft, Nucl. Phys. B 153, 141 (1979); Commun. Math. Phys. 81 (1981) 267; P. van Baal, Commun. Math. Phys. 94 (1984) 397; Z. Guralnik and S. Ramgoolam, Nucl. Phys. B 521, 129 (1998) arXiv:hep-th/9708089. 
[26] D. Mumford, "Tata Lectures on Theta, vol I, II, III," In Progress in Mathematics, Vol. 28 (1983) Birkhauser.

[27] I. Antoniadis, A. Kumar and B. Panda, arXiv:0904.0910 [hep-th].

[28] P. Di Vecchia, A. Liccardo, R. Marotta and F. Pezzella, arXiv:0810.5509 [hep-th].

[29] R. Russo and S. Sciuto, JHEP 0704, 030 (2007) arXiv:hep-th/0701292]; D. Duo, R. Russo and S. Sciuto, JHEP 0712, 042 (2007) [arXiv:0709.1805 [hep-th]].

[30] H. Abe, K. S. Choi, T. Kobayashi and H. Ohki, JHEP 0906, 080 (2009) arXiv:0903.3800 [hep-th]].

[31] D. Cremades, L. E. Ibanez and F. Marchesano, JHEP 0307 (2003) 038 arXiv:hep-th/0302105.

[32] T. Higaki, N. Kitazawa, T. Kobayashi and K. j. Takahashi, Phys. Rev. D 72 (2005) 086003 arXiv:hep-th/0504019.

[33] L. E. Ibanez, H. P. Nilles and F. Quevedo, Phys. Lett. B 192, 332 (1987).

[34] T. Kobayashi, Y. Omura and K. Yoshioka, Phys. Rev. D 78, 115006 (2008) arXiv:0809.3064 [hep-ph]]. 